\title{
Landesplanung als Studienobjekt an Mittelschulen
}

\author{
Oskar Bär, Werner Nigg, Jörg Roth-Kim, Zürich
}

Immer wieder liest man in der Presse von Landesplanung. Hie und da macht es den Anschein, als ob dieses Wort sogar als Propagandamittel für Personen und Parteien verwendet werde. Dabei ist eine sachliche Aufklärung unseres Volkes über Orts-, Regional- und Landesplanung heute notwendiger denn je. Bei dieser Aufklärungsarbeit fällt der Schule - vor allem der Mittelschule - eine wichtige und schöne Aufgabe zu. Am geeignetsten dazu ist die Geographie; denn erstens ist die Landschaft das eigentliche Untersuchungsobjekt sowohl der Geographie als auch der Landesplanung und zweitens sind beide - Geographie und Landesplanung - ausgesprochen interdisziplinäre Fächer.

Das Literargymnasium Zürichberg (Kantonsschule) führte vom 4. bis 8. Februar 1969 für die obersten beiden Klassen eine Studienwoche über Probleme der Landesplanung durch. Organisation und Leitung der Veranstaltung lagen in den Händen der Geographielehrer. Das Programm umfaßte folgende Hauptvorträge von Planungsfachleuten: Landesplanung als integrale Aufgabe (Prof. Dr. G. Grosjean, Bern) - Natürliche Grundlagen der Planung, Bevölkerungsentwicklung und Besiedlung (Prof. Dr. R. Meyer, Zürich) - Regionalplanung im Kt. Zürich (P. Schatt, Zürich) - Probleme und Methoden der Ortsplanung (Th. Stierli, Zürich) - Wie kam es zur Stadt? Was wird aus der Stadt? (Prof. Dr. Ernst Egli, Meilen). Eine ganztägige Exkursion ermöglichte den Schülern, in kleineren Gruppen die Planungsprobleme einiger Gemeinden mit recht unterschiedlichen Voraussetzungen zu studieren (Greifensee, Volketswil, Schöfflisdorf, Wetzikon, Meilen, Hittnau oder Regensdorf). Die nachher im Plenum vorgetragenen Referate über die Beobachtungen der einzelnen Exkursionsgruppen führten zu einer angeregten Diskussion am "Runden Tisch» über Grenzen der Planung und über Fragen des Bodenrechts (Leitung: Dr. R. Stüdeli, Zürich). Eine Halbtagesexkursion nach Baden-Dättwil veranschaulichte die Referate von A. Hentz und F. Wagner (Baden) über die Planung der Innenstadt von Baden und die Ortsplanung von Dättwil.

Wie ernsthaft sich die Schüler mit den Problemen auseinandersetzten, zeigten die treffenden Fragen und die inhaltsreichen Diskussionen, welche den Vorträgen folgten. In den abschließenden Berichten über die Studienwoche bekannte die Mehrzahl der Teilnehmer, daß ihnen die Probleme der Landes- planung während der Studienwoche zum ersten Mal bewußt gemacht worden seien. Sie zeigen sich von Umfang und Bedeutung der Aufgaben beeindruckt. Dieses Ergebnis macht deutlich, daß in den Schulen die Grundlagen für das Verständnis planerischer Arbeit gelegt werden können. Die Materie verlangt allerdings einen gewissen Grad geistiger Reife von Seiten der Schüler. Einen Erfolg können wir daher nur dann erwarten, wenn das Fach Geographie bis in die letzte Klasse vor der Matur unterrichtet wird.

An der Kantonalen Oberrealschule Zürich (Mathematisch-Naturwissenschaftliches Gymnasium) wurde 1963 der sogenannte Gruppenunterricht eingeführt, der den Schülern der beiden obersten Klassen Gelegenheit bietet, in einem frei gewählten Fach Kenntnisse über ein Stoffgebiet zu erwerben, welches im lehrplanmäßigen Unterricht nicht behandelt wird. Diesen Gruppenunterricht benützte ich für die Einführung in Probleme der Landesplanung. In diesem Jahr wird bereits der 7. Kurs durchgeführt ${ }^{1}$. Berufene Persönlichkeiten haben uns in verdankenswerter Weise mit Referaten und Führungen geholfen, so dipl. Architekt W. Aebli, H. Aregger, Dr. T. Hunziker, Prof. B. Hoesli, Forsting. J. Jacsman, Prof. R. Meyer, Prof. E. Winkler und weitere Fachleute. Unsere Kurse umfaßten jeweils drei Teile:

1. Einführung in die Probleme (Kurzreferate des Lehrers und der Schüler mit Diskussionen). Einmal habe ich gemeinsam mit den Schülern eine Schulfunksendung über Landesplanung zusammengestellt, die 1968 ausgestrahlt wurde.

2. Vorträge von Planern, Diskussionen und Exkursionen. Die Exkursionen führten u. a. durch die Stadt Zürich, nach Spreitenbach, ins Sihltal, nach Glattbrugg-Kloten, nach Dübendorf (Ausstellung über Ortsplanung), zu den Baustellen der N 3 und nach Bern (Altstadt, Bahnhof, Tscharner- und Halensiedlung).

3. Praktische Planungsarbeit. Die Schüler hatten in Fällanden und Witikon Erhebungen und Kartierungen in Richtpläne zu erarbeiten, wobei dank ihrer unbeschwerten Phantasie zum Teil originelle und gewagte Projekte entstanden. Höhepunkt und $\mathrm{Ab}$ schluß bildete jeweils die Beurteilung der Richtpläne durch bewährte Planer. Dabei kam es ge- 
wöhnlich zu interessanten und lehrreichen Diskussionen.

Allen Kollegen bieten sich während ihres Unterrichtes immer wieder Gelegenheiten, auf Probleme der Landesplanung einzutreten. Solche Arbeit «im Stillen» ist m. E. ebenso wertvoll wie großaufgezogene Kurse und Arbeitswochen.

Zur Landesplanungsausbildung von Geographen an der ETH

Seit 1943 besteht an der ETH ein Institut für Landesplanung, das anfänglich von Geographen betreut wurde. In Vorlesungen und - von Anfang an interdisziplinär gehaltenen - Ubungen wurden interessierte Absolventen der Hochschule (Agronomen, Architekten, Bauingenieure, Forstingenieure, Geographen, Kulturingenieure u. a.) mit der Orts-, Regional- und Landesplanung vertraut gemacht. 1961 wurde das Institut verselbständigt und der Unterricht in Landesplanung intensiviert. So können seither Geographen im Schlußdiplom eine Prüfung in ihr ablegen. In der Folge kamen die Behörden zur Überzeugung, daß die Ausbildung in diesem Fach durch ein Nachdiplomstudium noch weiter zu verbessern sei. 1967 wurde ein viersemestriger Kurs eingerichtet, zu welchem Absolventen schweizerischer und ausländischer Hochschulen mit Diplomen in Architektur, Bau- und Kulturingenieurwesen, Naturwissenschaften (Geographie), Land- und Forstwirtschaft, Rechtswissenschaft, Nationalökonomie und Soziologie zugelassen sind. Die Teilnehmerzahl ist beschränkt. Das Ziel der Ausbildung ist die Befähigung der Studierenden, als Spezialisten (ihres Faches) in Planungsteams zu arbeiten oder als Chef eines Planungsamtes zu wirken. In den ersten zwei Semestern werden sie daher in die ihnen nicht bekannten Fächer eingeführt; die nächsten Semester dienen der Vertiefung des Spezialgebietes und zudem der Schulung in Planungstechnik und Planungspraxis. Jeder Student hat selbständig eine Ortsplanung und interdisziplinär, d. h. in einer Gruppe eine Regionalplanung auszuführen. Unterrichtsfächer sind Landesplanung, Verkehrswesen, räumliche Gestaltung, Versorgung, Landschaftspflege, Land- und Forstwirtschaft, Kulturtechnik, Soziologie und Politologie, Volkswirtschaft, Sozialpsychologie, Siedlungshygiene, Mathematik, Statistik und Jurisprudenz. Die Studierenden erhalten den Status von Praktikanten Gebühren werden keine erhoben; andrerseits sind finanzielle Unterstützungen mög. lich. Im ersten Kurs (seit 1967) nahmen neben Architekten, Soziologen und Forstingenieuren drei Geographen teil. Weitere Orientierung gibt das Institut für Orts-, Regional- und Landesplanung der ETH, Weinbergstraße 89-100, 8006 Zürich.

\section{Anmerkung}

1 In Heft 3 der "Geographica Helvetica» 1964, ist ein Bericht über den ersten Kurs erschienen. 